\title{
Spanish ATLAS Tier-1 \& Tier-2 perspective on computing over the next years
}

\author{
Santiago González de la $\mathrm{Hoz}^{1}$, Carlos Acosta-Silva ${ }^{3,4}$, Javier Aparisi Pozo ${ }^{1}$, Manuel \\ Delfino $^{3,4}$, Jose del Peso ${ }^{2}$, Álvaro Fernández Casani ${ }^{1}$, José Flix Molina ${ }^{4,5}$, Esteban Fullana \\ Torregrosa ${ }^{1}$, Carlos García Montoro ${ }^{1}$, Julio Lozano Bahilo ${ }^{1}$, Almudena del Rocio Montiel $^{2}$, \\ Andreu Pacheco Pages ${ }^{3,4}$, Javier Sánchez Martínez ${ }^{1}$, José Salt ${ }^{1}$ and Aresh Vedaee ${ }^{3,4}$ on \\ behalf of the ATLAS Collaboration \\ ${ }^{1}$ Instituto de Física Corpuscular (IFIC), centro mixto CSIC - Universitat de València, Paterna, Spain \\ ${ }^{2}$ Departamento de Física Teórica y CIAFF, Universidad Autónoma de Madrid, Madrid, Spain \\ ${ }^{3}$ Institut de Física d'Altes Energies (IFAE), The Barcelona Institute of Science and Technology, \\ Campus UAB, 08193 Bellaterra (Barcelona), Spain \\ ${ }^{4}$ Port d'Informació Científica (PIC), Campus UAB, 08913 Bellaterra (Cerdanyola del Vallès), Spain \\ ${ }^{5}$ Centro de Investigaciones Energéticas, Medioambientales y Tecnológicas (Ciemat), Madrid, Spain
}

\begin{abstract}
Since the beginning of the WLCG Project the Spanish ATLAS computing centers have participated with reliable and stable resources as well as personnel for the ATLAS Collaboration. Our contribution to the ATLAS Tier2s and Tier1s computing resources (disk and CPUs) in the last 10 years has been around 4-5\%. In 2016 an international advisory committee recommended to revise our contribution according to the participation in the ATLAS experiment. With this scenario, we are optimizing the federation of three sites located in Barcelona, Madrid and Valencia, considering that the ATLAS collaboration has developed workflows and tools to flexibly use all the resources available to the collaboration, where the tiered structure is somehow vanishing. In this contribution, we would like to show the evolution and technical updates in the ATLAS Spanish Federated Tier2 and Tier1. Some developments we are involved in, like the Event Index project, as well as the use of opportunistic resources will be useful to reach our goal. We discuss the foreseen/proposed scenario towards a sustainable computing environment for the Spanish ATLAS community in the HL-LHC period.
\end{abstract}

\section{ATLAS Computing Challenges at HL-LHC period}

In the High Luminosity LHC period (HL-LHC) the ATLAS computing demands far outstrip the budget dedicated to computing by the different countries. On the other hand, physics analysis and simulation from ATLAS are already limited by the current computing resources. Therefore, new techniques have to be developed to enhance a robust network, a key-

\footnotetext{
*Corresponding author: santiago.gonzalez@ific.uv.es

(C) 2018 CERN for the benefit of the ATLAS Collaboration. CC-BY-4.0 license
} 
component in the WLCG (World Wide LHC Computing Grid) [1] architecture, minimize storage needs and make the most of opportunistic resources (HPCs) [2].

Assuming a flat budget for the facilities, a $20 \% / \mathrm{yr}$ capacity growth, and a constant development effort, $\mathrm{a} \sim 3 \mathrm{x}$ shortfall of CPU resources with respect to the flat budget model is estimated for the HL-LHC (Run 4), see Figure 1. In this case, the potential use of coprocessor/GPUs has not been considered.

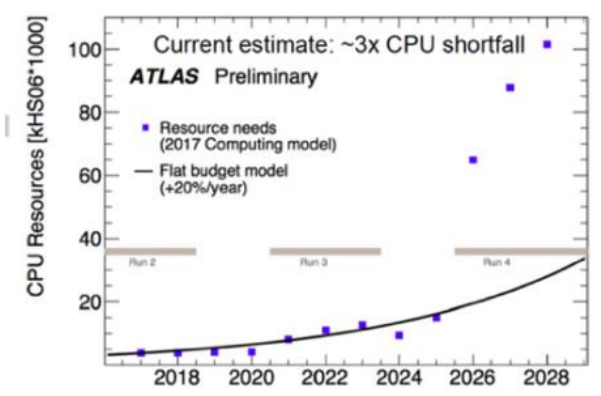

Fig. 1. Current estimate: $\sim 3 \mathrm{x}$ CPU shortfall [2].

In Figure 2, we can see $\sim 6 \mathrm{x}$ shortfall by today's estimate in Storage on disk. This is the ATLAS bigger problem because "opportunistic storage" basically does not exist. ATLAS collaboration is working on format size reductions, but it is hard to achieve large gains so ATLAS needs new approaches. A way to dramatically reduce the storage footprint is to increase the use of tape but tape access is slow and its use complicates the workflow.

Disk storage $\sim 6 \mathrm{x}$ short at HL-LHC

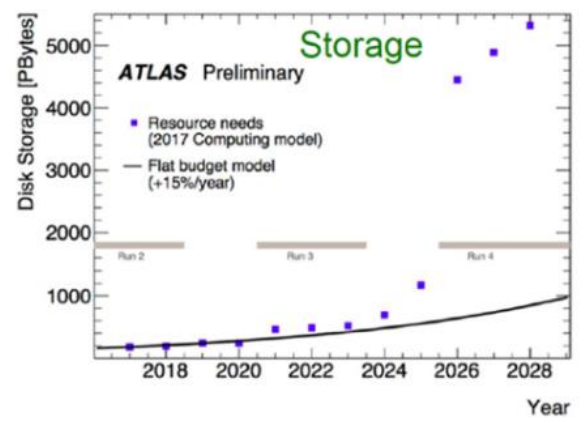

Fig. 2. Storage scaling to HL-LHC [2].

\section{Spanish ATLAS Tier-1 \& Tier-2 Federation}

The Spanish computing community is formed by one Tier-1 (PIC), and a federated Tier2: ES-ATLAS T2 (50\% IFIC, 25\% IFAE and 25\% UAM). So far, the community has provided the hardware resources fulfilling the ATLAS requirements of the Resource Review Board of the LHCC committee, contributing around $4 \%$ of the total resources of all ATLAS Tier-2s and $5 \%$ of all Tier-1s. These resources are integrated in the WLCG project and follow the ATLAS Computing Model. Present resources provided are shown in the Table 1:

Table 1. Hardware resources provided by the Spanish community in September 2018. 


\begin{tabular}{|c|c|c|c|}
\hline Site & $\begin{array}{c}\text { CPU (HEP- } \\
\text { SPEC06) }\end{array}$ & DISK (TB) & TAPES (TB) \\
\hline PIC-Tier1 & 46200 & 3265 & 9670 \\
\hline IFIC-Valencia & 26751 & 2146 & - \\
\hline IFAE-Barcelona & 10420 & 980 & - \\
\hline UAM-Madrid & 10358 & 1220 & - \\
\hline
\end{tabular}

In the recent LHC running periods around $6 \mathrm{~PB}$ of data have been stored in the Spanish facilities as shows the Figure 3 .

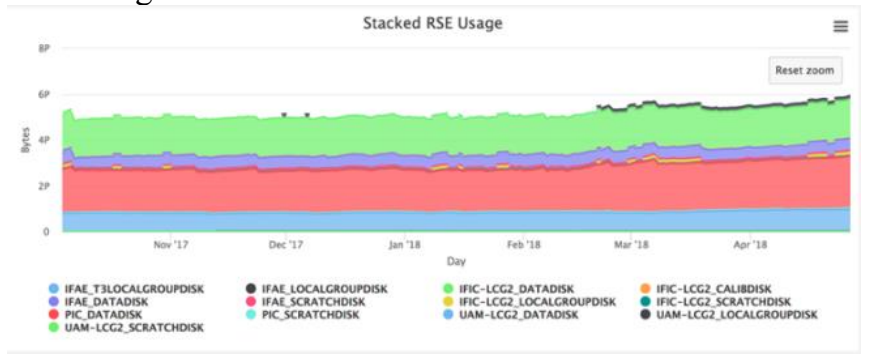

Fig. 3. Disk usage in the last 6 months in Spanish sites.

Concerning the facilities performance in terms of availability and reliability, Table 2 shows the values for the 2017 year. As can be seen, in both cases the average over all the Spanish sites is greater than $98 \%$.

Table 2. Site Availability and Reliability performance, based on WLCG information in the year 2017.

\begin{tabular}{|c|c|c|}
\hline Site & Availability (2017) & Reliability (2017) \\
\hline PIC-Tier1 & $98.96 \%$ & $99.22 \%$ \\
\hline IFIC-Valencia & $98.25 \%$ & $98.54 \%$ \\
\hline IFAE-Barcelona & $98.65 \%$ & $98.97 \%$ \\
\hline UAM-Madrid & $98.92 \%$ & $99.67 \%$ \\
\hline
\end{tabular}

\section{The Event Index (EI) Project}

The ATLAS EI project indexes and catalogues all events produced by ATLAS. This allows to rapidly locate individual events and study large quantities of data. It uses industry big data tools like Hadoop for storing properties of all real and simulated data, and additionally Oracle database storage for fast dataset discovery. In Figure 4, we can see the general architecture and data workflow of the ATLAS EI project.

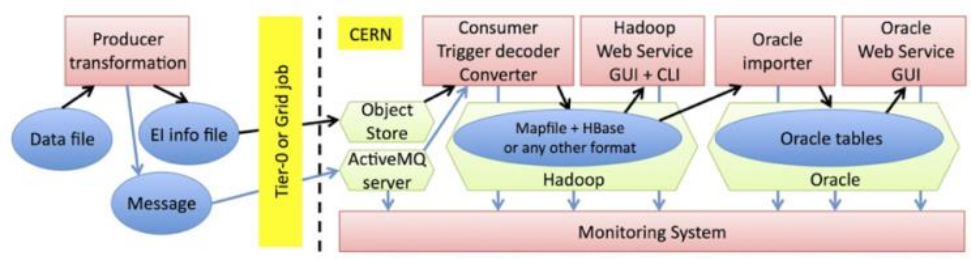

Fig. 4. General architecture and data workflow of the ATLAS EI project.

IFIC-Tier2 group is involved in several areas, starting with the Data Production in charge of indexing all valid data, and represented in the left side of Figure 4 with the Producer 
transformation entity that receives an input data file and produces a smaller EI metadata info file and control messages. IFIC group is also responsible for the architecture design and operation of the Distributed Data Collection, represented in next part of the Figure 4 and that since last year has put in production the new system based on an Object Store $[3,4]$ with good results. The vertical dashed line in the Figure 4 represents the boundary between the multiple distributed sites running the Producers, and the central services at CERN.

The control messages sent by Producer are collected centrally at CERN with the ActiveMQ Server, and the EI info file temporarily stored at Object Store. The EI Data Collection Supervisor is in charge of orchestrating the different components of the distributed system that indexes the datasets, and is the first that receives the control messages reading from ActiveMQ servers. The Consumer entity represented in Figure 4, retrieves the multiple EI info files that represent an indexed dataset from Object Store, and informs back the supervisor when the dataset has been stored in Hadoop. A web application provides information of the current state of the supervisor. Through this application, the progress of the datasets being indexed can be followed and corrective actions can be performed when needed.

The right side of Figure 4 represents the Hadoop and Oracle services where data is finally consolidated, and ready to serve users and services for data lookup and analytics.

With respect to the tools for analysing the data, the Trigger Counter is a web service that is able to provide meaningful information about the L1 and HLT triggers of a dataset in a human time scale by means of Hadoop MapReduce jobs which process a very compact and optimised representation of the triggers per dataset. Given a dataset and an optional filtering expression about the presence or absence of some triggers, and/or the value of lumiblock (information on the collection of luminosity blocks) and/or bunch id of the event, it can: 1) count the occurrences of each trigger; 2) compute the overlap between all the triggers, as is shown in the Figure $5 ; 3$ ) provide a limited list of the events that satisfy the provided expression. The service is able to provide interactive plots of the results as well as the data in JSON and CSV formats. The service is able to process millions of events and billions of triggers in a couple of minutes.

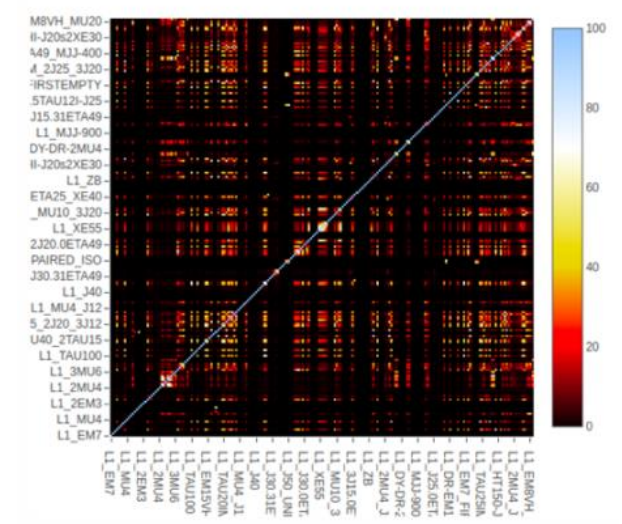

Fig. 5. A heatmap of the trigger overlaps of a dataset is shown.

\section{HPCs in Spain: Use of Extra Resources}

In 2017 and 2018 we were granted CPU hours in the RES (Spanish Supercomputing network). The RES allocated us 70k hours in the Lusitania supercomputer (based at CENITS) and two periods of 100k each in Mare Nostrum (based at BSC), which corresponds to using the complete PIC Tier-1 resources for about half a day. The Mare Nostrum has a 
peak performance of 11.15 Petaflops with 384.75 TB of main memory and 3,456 nodes (2x Intel Xeon Platinum $816024 \mathrm{C}$ at $2.1 \mathrm{GHz}$ ). Lusitania has $2 \mathrm{HP}$ Integrity SuperDome SX2000 with 64 Itanium 2 Montvale (1.6 GHz, 18 MB cache) processors each (128 processors / 256 cores).

ATLAS production workflow does not automatically send grid jobs to remote HPC systems; indeed, it is a hard task [5] and still under work in the community. Sites need to perform some manual work in order to be integrated and efficiently use HPC resources. Common constraints from HPC systems are: no access to the outside from the compute nodes, no root privileges, or not being able to install any system wide-software, no compatible Operating System in which we can re-compile the current ATLAS software. In this scenario, it is required to send grid jobs to the HPC and to run ATLAS simulation events upon request.

The solution for installing the ATLAS SW has been to request a Singularity [6] installation in the HPCs and transfer a complete file image of the ATLAS SW into the two supercomputers because they do not have CVMFS. The input (event generation) and output (Geant4 full simulation) processed files are transferred into and from the HPCs using ssh. Both IFIC and IFAE use ARC Computing Element [7] as interface between the ATLAS grid infrastructure and the remote HPC installed at IFAE and IFIC.

In May 2018 the two HPCs started to simulate events fully integrated in the ATLAS grid infrastructure. Figure 6 shows the activity during the peak week from the 18 th to the 25 th of May. The figure shows how the HPC multiplied the computing capacity of the Spanish ATLAS computing activity by up to a factor of 5 . The number of hours allocated to us was consumed in a few days. This exercise will be extremely valuable to assess the use of HPCs in High Energy Physics for the computing needs of the HL-LHC. The output is registered in the ATLAS catalog once it is at IFIC or at IFAE in a synchronous way.

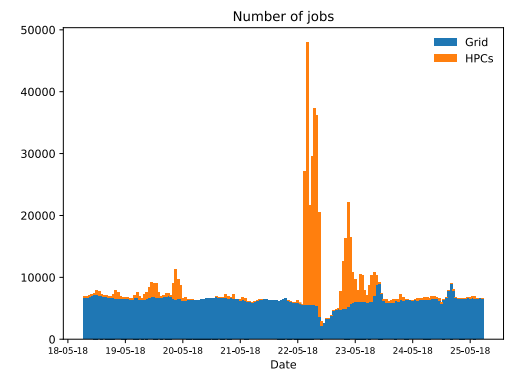

Fig. 6. Number of jobs running in Spanish Grid resources and Spanish HPCs from $18^{\text {th }}$ to $25^{\text {th }}$ May.

\section{Network LHCONE in Spain}

LHCONE [8] is a collaborative network implemented for the LHC research community as a controlled access "walled garden" routed IP internet. It improves the use of the network to facilitate data analysis, which is mainly done in Tier-2s. Specifically, this infrastructure improves the transfers between Tier-1s and Tier-2s and from Tier-2 to Tier-2. It complements LHCOPN, which accommodates transfers between Tier-0 and Tier-1s.

PIC and IFAE joined LHCONE in 2010, as soon as the infrastructure started operating. UAM has joined in 2017. It imposes a few guidelines to be followed to keep a symmetric routing, as well as some networking policies to provide a mutual trust among sites where only valid LHC traffic is routed. Such configurations are performed in the BGP (Border Gateway Protocol), both at NSP (Network Service Provider) and site ends. Figure 7 shows the performance for LHCONE at the UAM, PIC and IFAE. 


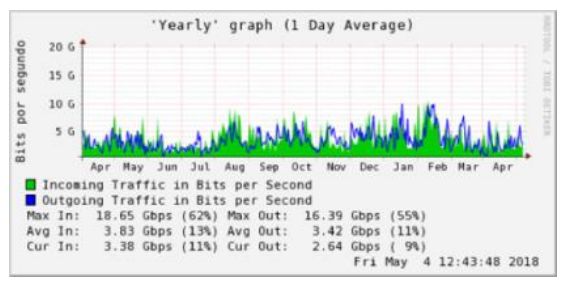

Fig. 7. Daily LHCONE traffic in RedIRIS in the last year (for PIC, IFAE \& UAM).

In particular, the UAM-LCG2 site joined whilst upgrading the network link. The link was increased up to $10 \mathrm{Gbps}$. In this new configuration, there is a second backup link, supplied by a different provider. The main 10Gbps line is connected to LHCONE through RedIris (Spanish academic and research network). The backup link is maintained by the University, and connected via CIEMAT to the outside. PIC Tier-1 and IFAE Tier-2 use a 20Gbps WAN network, connected to LHCONE at $10 \mathrm{Gbps}$.

At the site level, all Tier-2 services to be included in LHCONE had to be reorganized and aggregated into a single range of IPs, in order to minimize the LHCONE Prefixes, and to assure good performance of routing algorithms.

\section{Federation Storage}

The strong collaboration among Spanish ATLAS sites allows to explore ways of federating access to the storage endpoints that would be considered as a unique entry point. This idea of a regional storage federation is aligned with the WLCG Data Steering Group strategies to face the medium/long term Data Management challenges, i.e. the Run3 and HL-LHC.

The regional federation should meet the following requirements: scalability, performance, WAN access, redirection and fault tolerance.

A first attempt is being tested at the UAM with DynaFed [9], which is a tool that meets the needs. The plan is to integrate the rest of the sites into the tool and evaluate it.

On the other hand, Spanish sites are going to participate in the CERN Data Lakes project to federate storage and reduce costs (see section 8 ATLAS Core Computing). Spanish sites also contribute to the Eulake project, which is based on the EOS system to evaluate the potential of a new distributed storage solution to build Data Lakes [10].

\section{Data Analysis (DA)}

Data Analysis activity in our sites is carried out in close collaboration with the ATLAS Physicists, keeping substantial CPU resources for data analysis. In our sites, more and more frequently, Physics analysis will be performed using Machine Learning (ML) methods, that would imply the use of GPUs and much more memory than provided by ATLAS standard Worker Nodes. At the same time, users can do analysis using just their browser through tools like SWAN (Service for Web based ANalysis) [11].

The application of ML techniques has been generalized in the LHC experiments and there are already a whole set of programs, libraries, etc. that are being used for different analyses [12]. In particular, we are interested in importing ML methods and tools developed and implemented in generic environments / frameworks into the standard workflow used by an ATLAS analysis physicist: ANACONDA, TensorFlow, etc....

In order to check these methods and tools, a physics analysis case has been chosen: the search of $t \bar{t}$ resonances in ATLAS [13]. Our work has started from the datasets placed at the UCI 
machine repository [14] obtaining the first results from the application of several ML methods on the GRID infrastructures (IFIC Tier-2 \& Tier-3).

So far, we have made the calculations on conventional CPUs but the final goal is to streamline the whole process with the use of GPUs. The gain using GPUs will be really impressive and we are in the phase of acquiring a cluster of GPUs.

SWAN is a platform to perform interactive data analysis in the cloud. The original SWAN project was developed, implemented and deployed at CERN, so that its backend combines state-of-the-art software technologies with a set of existing IT services supported by CERN resources. Basically, this service works as follows: SWAN is hosted by some kind of cloud instance (CERN OpenStack [15] in the original project, and LUSTRE in the local implementation at IFIC) so the web portal and the user notebook servers run in a virtual environment. A Docker [16] container that is allocated in the virtualized infrastructure creates these environments. The web portal is based on the JupyterHub [17] technology. On the other hand, the web-based interface of SWAN is based on Jupyter [17] notebooks (which allow to combine code, text and plots in the same document). In general, users log in with their credentials and request the creation of their notebook server by means of the JupyterHub portal. The execution of notebooks is encapsulated in personalized Docker containers, all this performed in some cloud infrastructure. Users can perform, synchronize and share their analysis with the notebooks. Finally, when a Docker container is created, it's also linked to other resources such as software repositories and data storages so that all that is needed to perform any analysis is directly accessible from the personalized environment.

IFIC's effort to implement a SWAN platform was mainly focused on adapting this service to the IFIC environment. Currently the implementation is in a "proof-of-concept" stage so that the service works properly but the full platform setup is still underway. The SWAN service is not available to all of IFIC's users because of security limitations of the current setup.

\section{ATLAS Core Computing}

PIC/IFAE are involved in the Working Groups of the WLCG project to develop tools and services for the HEP Software Foundation [18].

They are participating in Data lakes project where the goal is to define the functionality needed to implement a federated data center concept that aims to reduce the operational cost of storage, and at the same time better manage network capacity, whilst maintaining the overall CPU efficiency. This would include the necessary qualities of service, and options for regionally distributed implementations, including the ability to flexibly respond to model changes in the balance between disk and tape. For that purpose, PIC/IFAE took part in a coordinated effort to review tape management and test tape performance across Tier-1 sites: namely, two tape stress tests were performed respectively around the 20th of July and August 2018, then their results and related remarks were presented to ATLAS and other site administrators. Besides reporting metrics, profiling storage capacity and revising storage settings to boost performance, the tests helped acquire a better understanding of errors, bottlenecks, and margins of improvements on the side of other intermediary grid services (like Rucio and FTS), and drawbacks affecting dCache sites (due to potential sources of miscommunication between dCache and FTS). This survey was a necessary first step within a wider strategy adopted by ATLAS to deeply revise its computing model, at least for what concerns the data processing part, soon to be re-implemented according to the so-called ATLAS Data Carousel model.

They are also contributing to the Computing Resource Information Catalogue (CRIC) task force [19]. It is an evolution of AGIS (i.e. ATLAS Grid Information System) aiming to provide WLCG topology description complemented with experiment-specific configuration to all 4 LHC experiments. So, as an information system CRIC is still experiment oriented, 
but no longer ATLAS specific. By design it will consume information from different information sources (GocDB, EGI, HTCondor, etc.) and for its bootstrap from BDII, OIM, SiteDB, etc. and will be accessed by different users (site administrators, experiment coordinators, robots, etc.). PIC/IFAE are mainly contributing to the implementation of various cron plug-ins to fetch data from different sources of information and also to the implementation of the authentication/authorization system based on different authentication methods (SSO, VOMS, SSL-certificate, etc.).

\section{Conclusions}

We have presented the evolution and technical updates in the ATLAS Spanish Federated Tier2 and Tier1. Spanish ATLAS Tier-1 \& Tier-2 infrastructure started in 2005 and it is offering $5 \%$ and $4 \%$ of all ATLAS Tier- $1 \&$ Tier- 2 resources respectively.

ATLAS will need a factor of 5 more resources at HL-LHC compared to today and flat budget and $+20 \%$ /year from technology evolution fills part of this gap. Within this scenario, storage looks to be the main challenge to address. The ATLAS Spanish LHC computing community is participating to establish the main guidelines for the next years (HL-LHC) and is moving towards common implementations: Data Management, HPC, Resource Federations, Machine/Deep learning facilities, and increasing the network bandwidth.

This work was partially supported by MICINN in Spain under grants FPA2016-75141-C2-1/2-R, and FPA2016-80994-C2-2-R, which include FEDER funds from the European Union.

\section{References}

1. WLCG project: http://wlcg.web.cern.ch/

2. T. Wenaus, D. Costanzo, A. Di Girolamo, How HL-LHC challenges inform workload management R\&D:An ATLAS view, (WLCG\&HSF Workshop, Naples, 2018)

3. Á. Fernández, S. González, Performance Improvements of an Event Index Distributed System. In Proceedings of 47th International Conference on Parallel Processing, (ICPP 2018, Oregon)

4. S. González, Distributed Data Collection for the ATLAS EventIndex Project. Proceedings of $23^{\text {rd }}$ International Conference on Computing in High Energy and Nuclear Physics, (CHEP 2018, Sofia)

5. S. Campana, Distributed Computing in LHC Run2, J.Phys:Conf. Ser. 664032005 (2015)

6. G. Kurtzer, Singularity:Scientific containers for mobility of compute. PLoS One12(5):7459 (2017)

7. M. Ellert et al., Advanced Resource Connector middleware for lightweight computational Grids. Future Generation Computer Systems 23 219-240 (2007)

8. http://lhcone.web.cern.ch

9. http://lcgdm.web.cern.ch/dynafed-dynamic-federation-project

10. X. Espinal, Data Lake R\&D, (Joint WLCG\&HSF Workshop, Naples, 2018)

11. D. Piparo, SWAN : a Service for interactive Analysis in the Cloud, CERN-OPEN-2016-005 (2016)

12. D. Rousseau, Machine Learning in HEP. Proceedings of $23^{\text {rd }}$ International Conference on Computing in High Energy and Nuclear Physics, (CHEP 2018, Sofia)

13. Searching for Exotic Particles in HEP with Deep Learning. Nature Commun., 5.4308 (2014)

14. http://archive.ics.uci.edu/ml/datasets/HEPMASS

15. OpenStack: http://www.openstack.org

16. Docker containers http://www.docker.com

17. JupyterHub portal: http://github.com/jupyter/jupyterhub and Jupyter notebools: http://jupyter.org

18. J. Albrech et al., A Roadmap for HEP Software and Computing R\&D for the 2020s, arXiv:1712.06982 [physics.comp-ph] (2017)

19. A. Anisenkov, AGIS evolution for other communities. Proceeding of $26^{\text {th }}$ International Symposium on Nuclear Electronics \& Computing, (NEC 2017, Montenegro) 